\title{
MÁLAGA HOY, LABORATORIO DE IDEAS PARA UNA CIUDAD SOÑADA: MEGALOMANÍA, PROYECTOS- ESTRELLA Y ARQUITECTURA DEL BUSINESS
}

\author{
Javier González Torres \\ Fundación Victoria (Málaga). Fundamentos e Historia del Arte \\ Universidad de Málaga. Dpto Historia del Arte
}

\section{Resumen}

Y tras la tempestad... jvuelve el ladrillo! Cuando las cifras macroeconómicas hablan del fin de la recesión y el comienzo de una nueva época expansiva, instituciones públicas y empresas privadas vuelven a proponer planteamientos arquitectónicos y a encabezar proyectos urbanísticos que, por mor de la crisis, quedaron paralizados. Málaga y su provincia no escapan a esos sueños de grandeza especulativos. La configuración estética de estas iniciativas, al margen de cuestiones políticas o medioambientales, permite ahondar en las raíces de sus planteamientos, desgranando influencias, derivaciones o perversiones. Proponemos un paseo virtual, crítico, por una cartografía malagueña que no existe pero que puede analizarse a través de esbozos, maquetas, presentaciones infográficas y memorias descriptivas. El objetivo es conocer si suponen una novedad o, por el contrario, no son más que 'cortinas de humo' que repiten formas, modos y apariencias de otros tiempos.

\section{Palabras-clave: CIUDAD SOÑADA; ARQUITECTURA; ARTE; ESTÉTICA; URBANISMO}

\author{
MÁLAGA TODAY, LABORATORY OF IDEAS \\ FOR A DREAM CITY: MEGALOMANIA, HIGH- \\ END PROJECTS AND BUSINESS ORIENTED \\ ARCHITECTURE
}

\section{Abstract}

And after the storm... the building resumes! As macroeconomic figures indicate the end of the recession and the beginning of a new period of expansion, public institutions and private companies once again initiate architectural propositions and lead urban projects that where left unfnished. Málaga and its province also succumb to these great architectural ambitions. Their esthetic configurations will make it possible to study in depth the roots of these projects, unveiling their infuences, derivations and perversions. We are offering a virtual tour of a Málaga that, although does not exist yet, can be analyzed through rough drafts, models, visual presentations and descriptive reports. Whether all of these should be regarded as innovative or treated as echoes of past times will be considered at the end of the study.

Keywords: DREAM CITY; ARCHITECTURE; ART; ESTHETIC; URBAN PLANNING

\footnotetext{
González Torres, Javier. "Málaga hoy, laboratorio de ideas para una ciudad soñada: Megalomanía, proyectos-estrella y arquitectura del business". AusArt 6 (1): 43-54. D0I: 10.1387/ausart.19444
}

\section{AUSART}


La benignidad del clima y las notables cualidades naturales-paisajísticas conforman el atractivo de la provincia de Málaga. La consolidación de la marca 'Costa del Sol' entronca históricamente con la llegada de la burguesía industrial, el crecimiento demográfico y la consiguiente modificación urbana. Novedades que se plasmaron a finales del siglo XVIII en la capital en el Paseo de la Alameda y el Palacio de la Aduana. ¿Son éstos dos hitos capaces de reordenar el trazado primigenio y dotar a la urbe de elementos diferenciadores? Sí; y ambos constituyen el inicio de una larga secuencia que, con desigual acierto, continúa hoy.

Un largo camino que desde esas referencias históricas ha forjado la identidad turística de este entorno al amparo de una serie de intervenciones entre las que descuellan las puramente especulativas. A veces, éstas enmascaran los problemas reales que devienen de la ausencia de planes urbanísticos concretos o el olvido del interés general en pro de la satisfacción partidista de determinadas ideas.

\section{EL AYER DE MÁLAGA Y SU PROVINCIA: DEL REFORMISMO BURGUÉS Y ' $L A$ ARQUITECTURA DEL RELAX' A LOS SUEÑOS DE GRANDEZA}

A esos aludidos primeros planteamientos le siguen otras dos actuaciones prolongadas hasta comienzos del siglo XX, -la calle Larios y el Paseo del Parque-, 'necesarios' por cuestiones funcionales, higiénicas y de comunicación que desdibujaron la ciudad histórica frente a la apabullante actualidad burguesa, copatrocinadora de las obras.

A partir de entonces, la pervivencia de formas decimonónicas eclécticas se convirtieron en seña identitaria y en lastre para nuevas ideas. Las epidérmicas ráfagas modernistas así como la influencia de los 'neos' regionalistas, coparon distintas actuaciones para dar paso tras la Guerra Civil a la tímida entrada de los elementos del Movimiento Moderno (Méndez 2012).

Superada la autarquía económica y en pleno desarrollismo, el turismo fue una poderosa fuente de ingresos. Bajo el mediático lema Spain is different y a lo 
largo de la carretera N-340, en Málaga se expandió la 'arquitectura del relax', donde el Estilo Internacional se hacía presente a través del ocio, el placer y la felicidad. Un retro-alimentado collage pop, excéntrico (Ramírez 1987), en el que las vacaciones se envolvieron de vanguardia (Méndez 2017). Así se definió un espíritu constructivo vernáculo conformado por hoteles, apartamentos y construcciones singulares (Morales 1982).

Una reglamentación incongruente, la supremacía de condicionantes económicos por encima de la disponibilidad de terrenos y el espectacular aumento de población fueron las causas que explicaron el 'cajón desastre' de finales de la década de 1980 (Galacho 2005). El 'todo vale' permitió que poderes fácticos e intereses particulares se unieran en pro de una supuesta singularidad del destino ${ }^{1}$. Las consecuencias se vivieron a partir de los siguientes años 90: un territorio polémico, configurado por y para el turismo, en el que el caos y la masificación provocaron una 'metástasis urbanística'.

\section{LA ACTUALIDAD. ¿FIN DE LA CRISIS? iVUELTA AL LADRILLO!}

Cuando las cifras macroeconómicas hablan hoy del fin de la recesión, instituciones públicas y empresas privadas vuelven a reincidir en comportamientos pasados. Este proceso de acción-reacción se argumenta a partir de criterios de esparcimiento social, en los que la planificación y la gestión pública abren camino a otros intereses al objeto de crear un mapa de infraestructuras únicas y equipamientos notables.

¿Es este un 'urbanismo inteligente', reflejo de valores culturales comunitarios, comprometido con su entorno y respetuoso con el pasado? Posiblemente no. Estos proyectos no devienen del place by desing en el que se combinan eco-diseño y acciones sociales. No son actuaciones de apropiación colectiva, ni de revitalización de solares abandonados, ni reutilización de complejos industriales para usos alternativos, propios del 'urbanismo adaptativo'.

Luego, ¿sigue vigente esa antigua fórmula que pensaba el territorio urbano como el tablero en el que establecer una sinergia expansiva que originó la 'burbuja' urbanística? Es bastante probable que sí. La megalomanía, los proyectos-estrella y la arquitectura del business se evidencian en iniciativas capa- 
ces de 'cautivar' a la opinión pública, 'vendiéndose' como 'emblemas' de una ciudad soñada/contemporánea.

Son criterios básicos para el ordenamiento territorial el predominio del interés público, el desarrollo sostenible y la cohesión social. Ello implica cambios profundos en la cultura de planificación y gestión territorial; una amplitud de horizontes que choca con la rentabilidad electoral cortoplacista y con el impacto por la degradación del suelo, la deforestación y destrucción de los ecosistemas naturales, la contaminación del aire o del agua, así como la acumulación de desechos.

¿Y los criterios artísticos? En un supuesto ideal, debieran establecerse en paralelo a las construcciones sostenibles como propuesta de habitabilidad, acceso y confort. Han pasado más de 20 años desde que el Museo Guggenheim Bilbao, proyectado por Frank Ghery, provocara un efecto contagioso. La conjugación del titanio, la piedra caliza y el vidrio dieron entonces forma y volumen a un proyecto global de transformación urbanística; una sinergia regeneradora que contagió la ciudad bilbaína. A menor escala, el procedimiento que permite insertar un edificio icónico/emblemático dentro de un programa de revisión de un enclave se propagó por distintas ciudades (Jencks 2005), aunque respondiese más a particulares egolatrías que a una visión de conjunto.

\section{CARTOGRAFÍA CRÍTICA DE PROPUESTAS ACTUALES}

Una consecuencia negativa de este planteamiento deviene de los edificios 'cortina'; su 'magnificencia' tapa la inequidad redistributiva, territorial y espacial de un barrio, condenado a un ostracismo marginal. Los ejemplos en Málaga se localizan en el mismo Centro y los antiguos arrabales, convertidos en el backstage de un teatro idealista donde en los últimos 15 años ha desaparecido uno de cada cuatro edificios centenarios (Ozomek 2017).

Parece que en estos últimos años, la conciencia colectiva y los planes de inversión pública han encontrado un camino entre lo urgente y lo importante que quizás acabe con la situación; cuestión que tiene por responsables a las administraciones públicas -Estado central, Junta de Andalucía y Ayuntamiento- y 
a promotoras e inmobiliarias. Cuentan siempre con el pasotismo endémico de la sociedad malagueña, cuyo laissez faire es consustancial a su ser.

El marco normativo vigente ${ }^{2}$ marca unos límites que en la práctica especulativa derivan hacia planteamientos contrapuestos. El hecho se visibiliza cuando determinadas empresas proceden a exponer proyectos que no tienen nada que ver con la restauración y rehabilitación patrimonial, sino que vienen a ofertar 'nuevos centros' singulares a partir de los anteriores.

De ahí que el objeto de este trabajo sea partir desde la investigación multidisciplinar metodológica para estudiar una amplia serie de proyectos que en estos últimos años parecen tomar cuerpo. Su lenguaje estético, al margen de cuestiones políticas o medioambientales, permite desgranar influencias, derivaciones o perversiones. Proponemos un paseo virtual, crítico, por la provincia malagueña a partir de esbozos, maquetas, presentaciones infográficas y avanzados proyectos. ¿Supondrán éstos una novedad, por el contrario, no son más que 'cortinas de humo' que ocultan formas, modos y apariencias manidas? A continuación, algunas pistas.

\subsection{OCIO Y DEPORTE: LA MODA DE LA CONTEMPORANEIDAD A PARTIR DEL TIPISMO Y LAS COSTUMBRES}

El way of live de los habitantes de un entorno está marcado por sus pasatiempos. Málaga presume de su afición por el 'deporte rey' nacional y su situación costera proporciona condiciones especiales para el deporte náutico.
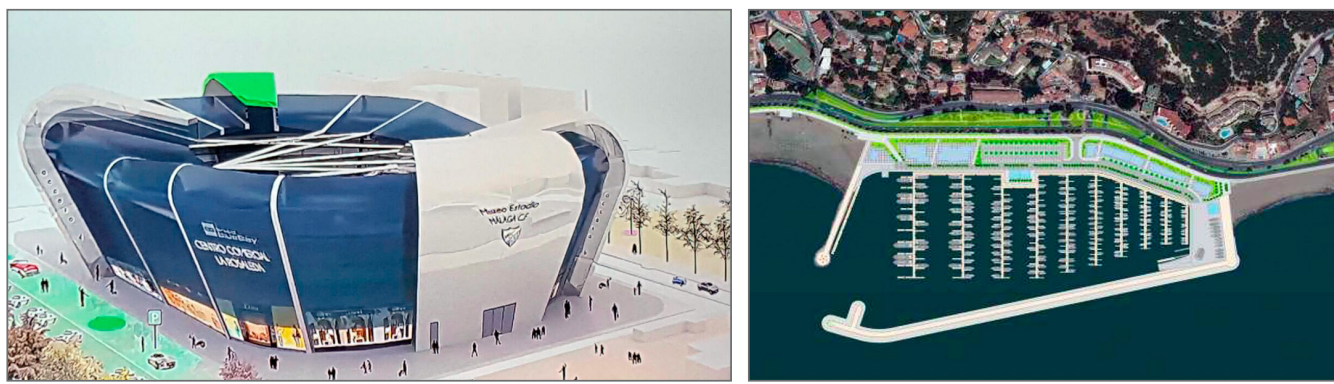

Imagen 1. La Rosaleda proyectada por Blue Bay y el Puerto deportivo de El Morlaco.

La Rosaleda es el estadio del Málaga CF. Las instalaciones de Martiricos han sido modificadas en varias ocasiones ${ }^{3}$ y sobre la misma se han planeado dos proyectos de remodelación integral. Uno de ellos es el presentado en 2016 por 
el grupo empresarial BlueBay ${ }^{4}$ y firmado por el estudio International Architects, apostando básicamente por recrecer las gradas para que de 27.000 localidades se pasen a 47.000. Como continuación de la estructura actual, se alzaría el nuevo graderío de sección oblicua, coronado por las habitaciones de un hotel de 160 habitaciones/cabinas. El entramado tectónico quedaría envuelto por una epidérmica capa exterior realizada con un material traslúcido que, a su vez, potenciaría sus tonos grises con la luz eléctrica del estadio. Aunque no sea un recinto cubierto, el alerón superior quedaría unido por unas tirantas centrales que equilibrarían pesos y tensiones.

Es indudable que la sombra del muniqués Allianz Arena es alargada ${ }^{5}$. Sin embargo, y a diferencia del referente pionero, el aspecto exterior del coliseo malagueño no sería precisamente 'escultórico' pues recordaría más a una barca hinchable. En todo caso, la actuación parece propia de un 'urbanismo mágico' pues la utilización del cauce del río como 'parking ecológico' conllevaría la realización de plan general para la ribera con múltiples actores.

La crisis y el impacto medioambiental frenaron la creación de distintos puertos deportivos. El previsto en El Morlaco, aledaño a los Baños del Carmen de la capital, ocuparía una superficie de $164.373 \mathrm{~m}^{2}$ entre el dique existente en La Caleta y uno de nueva construcción al oeste, con forma de 'L'. Con 485 puntos de atraque, el acceso se realizaría a través de 10 pantalanes decrecientes. En la línea de la costa, además de un aparcamiento, varios accesos peatonales lo conectarían al paseo marítimo si bien, en esencia, la propuesta no incluye elementos novedosos. Su ejecución, incluida en el Plan Director de Puertos de Andalucía (2014-2020) y aprobado por la Junta autonómica, queda condicionada -además de por su viabilidad económica- por la no construcción de otro muelle deportivo en San Andrés y por una actuación garantista de los valores marinos del entorno. En este sentido, quizás sea más adecuado la realización de un proyecto integral que salvaguarde el tramo comprendido entre La Malagueta y el comienzo de Pedregalejos y que contribuya a su mantenimiento más allá de las costosas obras de regeneración que se hacen, cada año, cuando los temporales 'se llevan' la arena de la playa.

\subsection{Estancias de lujo y confort: Entre la piqueta y la suge- RENTE CREACIÓN}

La potenciación de la capital como destino cultural y turístico lleva aparejado el aumento de instalaciones hoteleras. El número de camas/pernoctaciones ha crecido de manera considerable, convirtiéndose Málaga en alternativa a 
las cadenas de la costa al ofertar espacios de lujo y confort ${ }^{6}$. En este sentido, hay dos proyectos que comparten estos criterios, aunque sus planteamientos arquitectónicos sean diferentes.
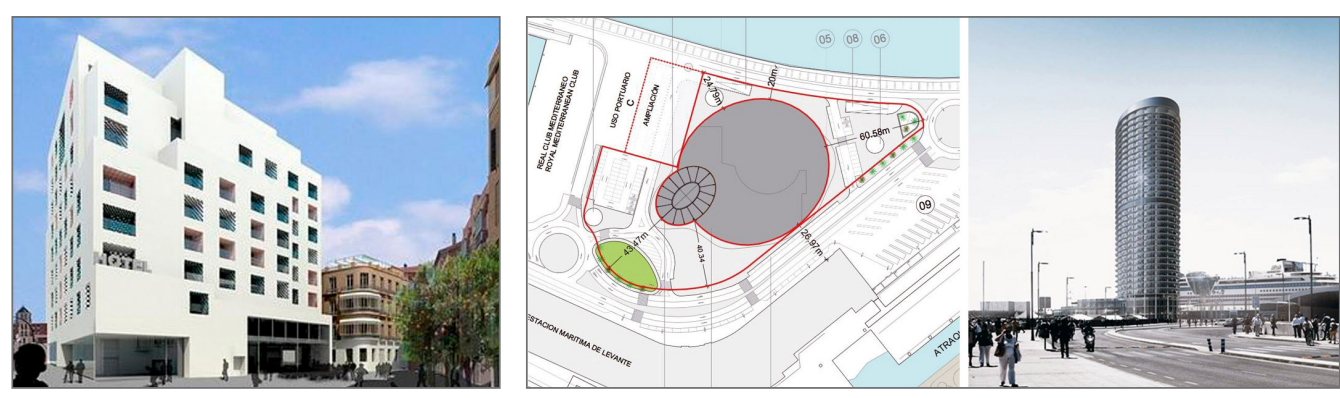

Imagen 2. Hotel proyectado por Moneo con la reproducción, al fondo, de La Mundial. Al lado, planta y alzado del Hotel Suites Málaga Port.

Por un lado, la unión temporal Braser pretende actuar en la zona de Hoyo Espartero/Atarazanas, linderos al norte de la Alameda principal, junto al Guadalmedina. Un hotel junto a dos bloques de oficinas y la urbanización del entorno constituyen el proyecto. Para ello demolerían dos manzanas de viviendas del siglo XIX -las de la zona oeste, en el Pasillo de Atocha, sufrieron ya los efectos de la piqueta-, proponiendo un conjunto que cuenta como principal aval la firma de su autor: Rafael Moneo. El edificio más voluminoso es el hotelero, un módulo rectangular de 10 alturas del que llama la atención la tonalidad blanquecina de sus paramentos murarios y su linealidad desornamentada 'pseudo-cenobial'. Matices que no comportan ningún rasgo de novedad en la obra del premiado autor, capaz de argumentar propuestas/conjuntos mucho más interesantes. A ello se le añade la demolición del palacete de los condes de Benahavís; un edificio de $800 \mathrm{~m}^{2}$ diseñado por Eduardo Strachan Viana-Cárdenas y que ha tenido una azarosa 'vida' desde su construcción en 1894. El valor tipológico del edificio y la singularidad de los cierres curvos de su esquina redondeada, con forja original, son razones más que fundamentales para su conservación. A cambio, la empresa constructora plantea la reproducción del mismo a modo de falsa operación 'de memoria conservativa', ubicándolo en el córner norte de la parcela. ¿Restauración y puesta en valor del patrimonio autóctono o pseudo-copia para engrandecer a su vez otro diseño más 'actual'?

El dique de Levante es un espacio de 614 metros de longitud, inaugurado en 2001. El Ayuntamiento de la ciudad y la Autoridad Portuaria han mostrado 
reiteradamente su predisposición por dotarlo de un edificio singular. En 2016 convocan un concurso público, presentándose con antelación un proyecto respaldado por la empresa Andalussian Hospitality II, denominado Hotel Suites Málaga Port. El estudio de José Seguí es el responsable del diseño del conjunto, compuesto por centro de congresos, zona comercial, piscinas y una aerodinámica torre elíptica de 135 metros de altura, 35 plantas y 352 habitaciones. El pormenorizado dossier explicativo del proyecto ${ }^{7}$ se centra en aspectos confortables y de eficiencia energética, argumentados para conferir la imagen final de 'hito arquitectónico singular'. Con ello no solo se atraerían cruceristas de alto standing, propios de los 'hoteles de destino', sino que se convertiría en elemento referencial del paisaje urbano. La fórmula apenas presenta novedades pues las soluciones estéticas y su emplazamiento hacen referencia a uno de esos emblemas que marcó la historia constructiva contemporánea: el hotel nacional Río de Janeiro que Oscar Niemeyer diseñase a finales de la década de 1960 para el barrio de São Conrado, en la capital carioca (Williams 2009). El vidrio, el aluminio y el hormigón fueron entonces los materiales empleados en una construcción planificada para 55 plantas -al final, solo 34-. Características que, salvando las distancias, se reinterpretarían de nuevo en Málaga. Como diría Ambrose Bierce, "no hay nada nuevo bajo el sol...".

\subsection{El nUeVO SKyline Residencial: LA EXCLUSividad de Vivir EN UN RASCACIELOS}

La máxima expresión de un sueño, el de conquistar el cielo; una inquietud que ha acompañado al ser humano desde el inicio de los tiempos, sigue siendo hoy, para algunos, un reto. En estos últimos años, hasta tres proyectos intentan desarrollar ese ansia en Málaga: las torres de La Térmica, las de Comarex y las de Martiricos. La primera, promovida desde 2006 por Reyal Urbis con diseño de Carlos Ferrater, al oeste de la ciudad, en la prolongación del paseo Antonio Banderas, sobre los solares de una antigua central eléctrica; la segunda, una propuesta de Luis Alonso y Sergio Balaguer proyectada también en 2006 y modificada después sobre los terrenos de los antiguos depósitos de Repsol, una parcela de más de $177.000 \mathrm{~m}^{2}$ que separa los distritos de Carretera de Cádiz y Cruz del Humilladero; y, la tercera, diseñada en 2015 por Dorronsoro Arquitectos para la promotora Espacio Medina, para edificar en dos parcelas contiguas -la explanada de Martiricos y los terrenos de la antigua fábrica de Citesa-.

Es este último proyecto el que más avanzado se encuentra, habiéndose comenzado la cimentación del edificio de viviendas que, con forma de ' $U$ ', 
se alza al sur del terreno. Los otros dos viven situaciones distintas: en el del paseo marítimo, los derechos edificatorios pasaron a la Sareb, transfiriéndose recientemente al grupo Metrovacesa; el de los terrenos de la industria energética está en suspenso tanto por la quiebra de la promotora como por la falta de consenso municipal, unido a la posible contaminación de los suelos. De todas formas, los tres proyectos cuentan con un planteamiento integral de renovación urbanística, uniéndose a las torres-rascacielos otros equipamientos, espacios públicos y amplias zonas verdes.
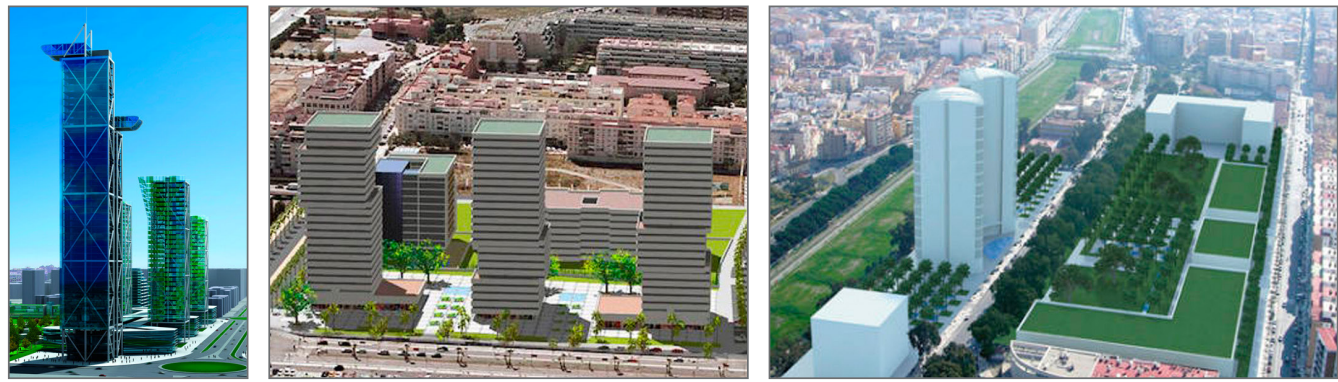

Imagen 3 (apartado 3.3). Las torres y sus conjuntos proyectados en Repsol, La Térmica y Martiricos. En este último, solo se aprecia el volumen genérico de las dos estructuras gemelas, sin que se especifiquen sus detalles estructurales ni arquitectónicos.

El skyline de Málaga quedaría modificado por el proyecto de Comarex: cuatro torres de 117, 124, 131 y 186 metros respectivamente, alzadas sobre un zócalo de dos plantas. Sus fachadas contarían con un doble revestimiento de vidrio, con los exteriores en tonos verdes; un juego de luces/reflejos que el propio estudio arquitectónico ya ha planteado en otras propuestas ${ }^{8}$, siguiendo las experimentaciones de Matthias Sauerbruch y Louisa Hutton.

Las tres torres de La Térmica rondarían los 130 metros, doblando así la longitud de la chimenea de la vetusta central energética. Compartiendo un mismo diseño de base cuadrada, sus 27 pisos destacan por el alzado quebrado, las tonalidades grises y una impronta asimétrica. Se pretende así potenciar el carácter escultórico final al objeto de romper la monotonía del resto del frente litoral ${ }^{9}$. La solución propuesta, repetida en otras ciudades, no reporta novedad alguna.

Finalmente, las Torres de Martiricos son aún una incógnita ${ }^{10}$. Su configuración final podría resultar de la colaboración entre Dorronsoro y el refutado estudio neoyorkino de Pei Cobb Freed \& Partners. Sólo se conoce que ambas edificaciones serían gemelas, tendrían una planta triangular con los vértices 
redondeados y que, a lo largo de sus 29 plantas -125 metros de altura total-, se dispondrían 464 viviendas.

\section{CONCLUSIONES}

Superado el provincianismo de otras épocas, Málaga se parece hoy a aquella urbe que preconizaba Sabina en La ciudad de neón y que cantaban en 1987 Javier Gurruchaga y la Orquesta Mondragón. La proyección internacional de una localidad debe estar centrada en admirar su particular memoria, reconociendo los rasgos propios de su identidad para proyectarlos y adaptarlos a un futuro donde el urbanismo juegue un papel respetuoso, pero abierto a novedosas y sostenibles propuestas. Parafraseando la letra, la vida que destilan los habitantes de Málaga y la Costa del Sol provoca el rechazo de las propias urbes, aquellas "que ha[n] crecido de espaldas al suelo", a la propia humanidad, convirtiéndose en sugerente "cárcel" capaz de ofrecer al visitante "que llega [...] un caramelo // con el veneno de la ansiedad". La despersonalización de los núcleos urbanos, su conversión en destinos turísticos de mercado donde se niega lo autóctono y se acoge a lo foráneo como propio, termina por ofrecer un "mapa de la soledad", un llamativo entramado de volúmenes arquitectónicos y supuestos espacios de esparcimiento ciudadano que, en realidad, terminan siendo un caricaturesco "ogro con dientes de oro".

La reconversión turística de todo Centro histórico en 'parque temático' es un mal cuasi endémico en Europa. El cambio de actividades económicas y la monotematización del espacio comunitario conlleva la pérdida de residentes y el aumento considerable de procesos gentrificadores. Basta un solo ejemplo en Málaga: la destrucción de los restos de la judería para la construcción del flamante Museo Picasso. La articulación de políticas de reversión de esta tendencia junto a una apuesta integradora mucho más decidida por la cultura, debieran centrar las miras de las instituciones públicas con tal de contagiar, concienciar y movilizar a la iniciativa privada y a la propia ciudadanía.

El debate sobre el modelo de ciudad también conlleva el estudio de la urbe vertical. De un lado, cuestiones de sostenibilidad y aprovechamiento; de otro, de especulación urbanística. La mezcla de ambas, a finales del siglo $X X$, fue una auténtica aberración. El Plan Estratégico de Málaga apostaba entonces por una ciudad turístico-tecnológica; pero el PTA se quedó aislado en las cer- 
canías de la barriada de Campanillas y su poder contagioso no se ha sabido extender al resto de la urbe ${ }^{11}$. La apuesta actual, rebautizada con el pomposo nombre de Smart City, pretende materializar aquello que fue imposible entonces. Veremos con el tiempo si se trata de un hecho firme o, por el contrario, se terminará desvaneciendo como las pompas de jabón.

Las innovaciones emergentes y el aumento de la conectividad a internet deben servir como acicates para corregir los problemas de la ciudad actual: la emisión de gases 'efecto invernadero', el aumento de la delincuencia y los altos costes energéticos lastran el panorama. ¿Será la Málaga del futuro una ciudad habitable, tendente hacia lo renovable, lo verde, lo humano, alejada de aquellos modelos especulativos de antaño? Solo el tiempo lo dirá. Mientras tanto, algunos seguirán soñando con una urbe plagada de supuestos 'iconos' que pretenden subvertir, empañar y/o destruir su milenaria existencia, originada en el prehistórico cerro de los Millares y convertida en prolífica factoría fenicia.

\section{Referencias}

Galacho Jiménez, Federico Benjamín. 2005. "El planeamiento urbanístico municipal de la Costa del Sol: Procesos de formulación y criterios de ordenación”. Baetica 27: 97-114

Jencks, Charles. 2005. The iconic building: The power of enigma. New York: Rizzoli

Morales Folguera, José Miguel. 1982. La arquitectura del ocio en la Costa del Sol. Málaga: Universidad de Málaga

Méndez Baiges, Maite, ed. 2012. Arquitectura, ciudad y territorio en Málaga (1900-2011). Málaga: Geometría

— . 2017. "El estilo del relax y la imagen pop de la Costa del Sol". En Immaginare il Mediterraneo: Architettura, arti, fotografia, a cura di Andrea Maglio, Fabio Mangone \& Antonio Pizza, v. 4, 101-10. Napoli: Art Studio Paparo

Ozomek Fernández, Antón Iván. 2017. "Degradación del Patrimonio". Gente corriente (blog), 10 abr. https://gentecorriente.net/blog/2017/04/10/degradacion-del-patrimonio/

Ramírez Domínguez, Juan Antonio. 1987. El estilo del relax: N-340, Málaga, h. 1953-1965. Una idea de Diego Santos, con fotografías de Carlos Canal. Málaga: Colegio de Arquitectos

Williams, Richard J. 2009. Brazil: Modern arquitectures in history. London: Reaktion

Notas

${ }^{1}$ Basta señalar, a modo de simples ejemplos, los proyectos publicitados por el alcalde de Marbella, Jesús Gil y Gil (1991-2002), cada vez que se presentaba junto a su partido a unas elecciones municipales: una isla artificial frente a Puerto Banús, un tren monorraíl o, incluso, un mega-aeropuerto. A ello debe unirse cómo años después, superados los casos 
de corrupción vividos por el Consistorio marbellí, se llegaron a proponer nuevos proyectos 'faraónicos' que entroncan con el espíritu de los citados y que, por distintas circunstancias, no se concretaron: la ampliación del Puerto de la Bajadilla, promovido por la empresa catarí Nasir Bin Abdullah \& sons bajo diseño del Estudio de José Seguí, en colaboración con J. M. Berenguer y F. Rueda (2008); y la Torre de Sierra Blanca, un rascacielos esbozado por Teodoro Cabrilla que pone en marcha la inmobiliaria Sierra Blanca Estates (2013).

${ }^{2}$ Ley de Patrimonio Histórico, su homónima andaluza y el PGOU-PEPRI local.

${ }^{3}$ Las dos de mayor calado se cifran: en 1982, con motivo de su conversión en sede del Campeonato Mundial de Fútbol, celebrado en España; y en el período 2000-2006, para adecuarse a la normativa internacional en materia de seguridad en instalaciones deportivas.

${ }^{4}$ La empresa hotelera mantiene un litigio sub iudice con el propietario del club a cuenta de una supuesta venta del $49 \%$ de las acciones de la sociedad en 2013.

${ }^{5}$ Sirva como cita la remodelación que Boogertman Urban Edge \& Partners realizan en 2010 en el Soccer Ctiy Stadium, de Johannesburgo, reinterpretando en sus frentes un particular elemento de la cultura del lugar: una olla de cerámica africana.

${ }^{6}$ Es cuanto menos curioso que los dos únicos hoteles de 5 estrellas de la ciudad sean de reciente apertura: el Vincci Selección Posada del Patio (2010) y el Miramar (2017). En ambos casos, se han rehabilitado inmuebles anteriores siguiéndose criterios diversos.

${ }^{7}$ Documentación al completo alojada en la web de la empresa promotora https://torrepuertomalaga.com

${ }^{8}$ Véanse edificios diseñados por la misma firma para Santiago de Chile, Barranquilla (México) y Tegucigalpa (Honduras).

${ }^{9}$ El diario Malaga Hoy, 4.08.2006, recoge unas interesantes consideraciones de Carlos Ferrater, autor del diseño arquitectónico.

${ }^{10}$ Véase la web http://www.parquemartiricos.com/

${ }^{11}$ Según un estudio de la consultora Infyde (marzo de 2018), la tecnópolis absorbe el 20,06\% del empleo de Málaga, suponiendo un impacto económico del $19,3 \%$ en el PIB metropolitano.

(Artículo recibido 14-03-18; aceptado 18-05-18) 